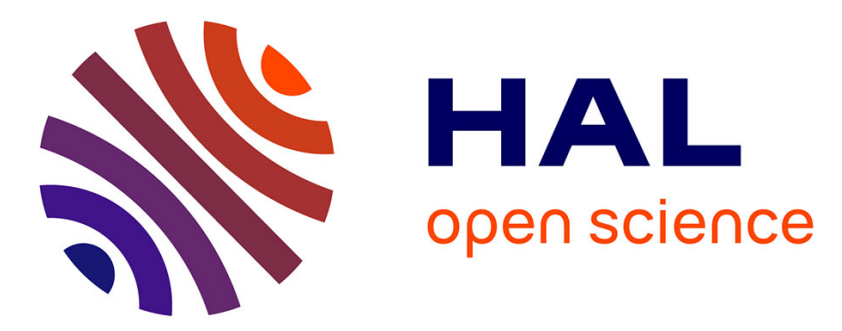

\title{
LES PARENTS DES CITÉS : LA PRÉVENTION FAMILIALE DES RISQUES ENCOURUS PAR LES ENFANTS
}

Catherine Delcroix

\section{- To cite this version:}

Catherine Delcroix. LES PARENTS DES CITÉS : LA PRÉVENTION FAMILIALE DES RISQUES ENCOURUS PAR LES ENFANTS. Les Annales de la Recherche Urbaine, 1999, AU RISQUE DES ESPACES PUBLICS, 83-84, pp.97-107. hal-01296746

\section{HAL Id: hal-01296746 https://hal.science/hal-01296746}

Submitted on 5 Apr 2016

HAL is a multi-disciplinary open access archive for the deposit and dissemination of scientific research documents, whether they are published or not. The documents may come from teaching and research institutions in France or abroad, or from public or private research centers.
L'archive ouverte pluridisciplinaire HAL, est destinée au dépôt et à la diffusion de documents scientifiques de niveau recherche, publiés ou non, émanant des établissements d'enseignement et de recherche français ou étrangers, des laboratoires publics ou privés. 


\section{Catherine Delcroix}

\section{LES PARENTS DES CITÉS :}

\section{LA PRÉVENTION FAMILIALE DES RISQUES ENCOURUS PAR LES ENFANTS}

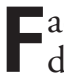
ce à la montée des incivilités des jeunes vis-à-vis des personnes plus âgées ou des représentants des institutions publiques et d'autres gestes plus violents ou plus spectaculaires, la mise en accusation de l'action éducative des parents de milieux populaires et en particulier des pères est systématique. Elle donne les moyens à beaucoup de responsables politiques, de professionnels de l'éducation, à des travailleurs sociaux et même à un certain nombre de sociologues d'expliquer une situation qui dérange, et insécurise.

"Aux adolescents violents, c'est-à-dire désespérés, font face des parents silencieux, parce que brisés et désemparés. L'autorité familiale est donc en berne et les enfants de ce fait bernés. Les familles des quartiers (économiquement) sinistrés n’ont plus la force de donner, de transmettre. Alors, quand la maison n'est plus qu'un mur de silence, l'appel de la rue se fait pressant avec tous les dangers et toutes les tentations que l'on sait ».

D'autres auteurs vont encore plus loin dans leurs analyses des comportements des jeunes, ils abordent le rôle parental en remettant en cause jusqu'au mode de transmission des valeurs.

«En matière de violence physique, la transmission familiale des normes de comportement obéit à des procédures explicatives et implicites qui se trouvent souvent en parfaite contradiction les unes avec les autres. D'un côté, les parents interdisent globalement et de manière formelle à leurs enfants de se battre dans la rue et à l'école et ils les punissent volontiers quand ils transgressent cet interdit; de l'autre, ils leur offrent des modèles de conduite et de pratique qui s'opposent parfois en tout point aux règles qu'ils sont censés vouloir transmettre $» .^{2}$

On pense alors pouvoir réduire la violence des plus jeunes en punissant les parents, en les privant notamment d'allocations familiales. Plutôt que d'être toujours à la recherche d'un coupable, il nous semble plus important de mettre à jour le rapport social ${ }^{3}$ qui transforme les différences sociales en inégalités scolaires, économiques, politiques, et ethniques et qui favorise la mise en place d'un processus d'entrée dans des comportements violents de la part de ces jeunes.
Il existe chez les décideurs des institutions françaises un doute sur les capacités de ces parents à faire respecter leur autorité par leurs enfants; c'est presque devenu un lieu commun. On n'en finirait pas de citer tous les articles de journaux, les rapports de

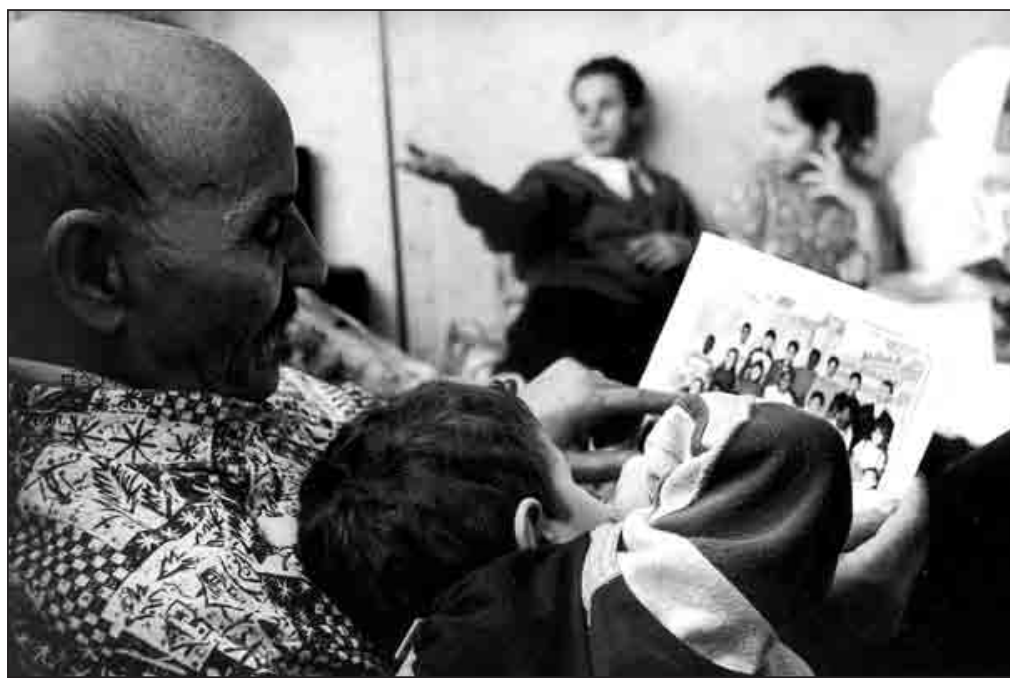

La Courneuve, cité des 4000.

recherche sur les "banlieues", qui donnent de ces parents, en particulier immigrés, une image dévalorisée. Parce que ces pères seraient ouvriers au chômage, RMIstes, ces mères isolées dans la monoparentalité ou alors enfermées chez elles sans lien avec la société, ces parents seraient donc "socialement invalidés "(l'expression vient de sociologues), voire "murés dans le silence», ils seraient incapables d'exercer leurs fonctions parentales :

1. Fize M., «L'autorité maltraitée... et revendiquée », Paris, Migrants-Formation $\mathrm{n}^{\circ} 112$, mars 1998, 20-25.

2. Lepoutre D., Cour de banlieue - Écoles, rites et langage, Paris, Odile Jacob, 1997

3. Cet objectif a été poursuivi avec succès par Alain Léger et Maryse Tripier qui ont réussi à mettre à jour le rapport social qui transforme les différences sociales en inégalités scolaires dans leur livre Fuir ou construire l'école populaire, Paris, Méridiens-Klincksieck, 1988.

Les Annales de la Recherche Urbaine $n^{\circ}$ 83-84, 0180-930-IX-99/83-84/p. 97-107 (C) METL. 
«Un nombre croissant de familles de ces quartiers vit grâce aux aides sociales diverses, certains jeunes bénéficient de quelques stages... Le travail comme forme principale d'accès au revenu et à la consommation, n'est déjà plus la norme, et les enfants grandissent dans un univers où l'effort individuel relève de l'héroïsme.... ${ }^{4}$.

Or au cours d'une recherche ${ }^{5}$ qui nous a amenée pendant cinq ans à étudier "les enjeux prioritaires et les types de conduites des familles populaires face à la précarité", ce ne sont pas des parents démissionnaires que nous avons rencontrés. Nous avons enquêté auprès de trente familles d'une cité HLM de Toulouse .

Nous avons cherché à décrire leurs situations, leurs parcours, leurs logiques d'action et leurs objectifs à long terme. Certes certains de leurs enfants ont, parfois, des comportements déviants; mais les parents cherchent à prévenir ce type de conduite et mobilisent à cet effet les ressources dont ils disposent malgré des environnements agressifs et discriminatoires vis-à-vis de leurs enfants.

D'emblée prenons l'exemple de Monsieur Kamel. Il est originaire de Tunisie; chauffeur de poids lourds, il a six enfants (les quatre premiers sont des filles). Il emmène à tour de rôle chacun de ses enfants dès l'âge de huit ans sur les routes de France. Chacune à leur tour, plusieurs fois dans l'année, les deux filles que nous avons interrogées partent découvrir avec leur père des villes, des monuments historiques. Elles rencontrent aussi des collègues de leur père. Voici comment elles racontent ces moments privilégiés :

Celle de 19 ans :

"Je vais avec lui pour visiter les villes de France et des places touristiques. On fait plusieurs pauses. Il me parle de l'histoire de ces lieux. Je compare (les villes) entre elles : avec Toulouse mais aussi avec Tunis, sa ville d'origine, et (avec) celles que nous visitons. Il montre les avantages et les inconvénients de chacun des endroits où nous allons. Je crois que c'est la curiosité qu'il m'a apprise, qui m’a poussée à apprendre les langues étrangères.»

Cette jeune fille est actuellement en deuxième année d'université. Elle étudie l'allemand et l'anglais. La fille de 16 ans est sensible à d'autres éléments :

"On fait la route ensemble; il me montre comment on conduit et on répare un poids lourd. Je vois comment il met le frein à main. J'ai envie de conduire, d'avoir un permis. Je suis en troisième; j'ai envie de passer un BEP Services Transports et Communication. Je voudrais passer un baccalauréat "économique et social» et ensuite créer une entreprise de transport routier; pas ici en France, mais en Tunisie. J'adore ce pays; même si je me sens bien ici aussi.»
Les témoignages de ces filles montrent comment M. Kamel ouvre à ses enfants plusieurs champs de possibles; comment il leur transmet une manière de regarder le monde en s'appuyant sur ses expériences de migration et de travail, qui ont pour avantage de permettre des comparaisons. Il favorise également l'apprentissage par ses enfants de codes appartenant à divers mondes culturels et sociaux. De plus il construit avec chacun d'eux, de façon très moderne, une relation directe qui ne passe pas par la mère. Dans cette famille les deux jeunes filles interrogées ont une connaissance précise de l'enfance et du passé de leur père. Elles savent pourquoi il a émigré et avec quel projet.

Sur le terrain, et en menant avec ces femmes, ces hommes et leurs enfants de nombreux entretiens, ce qui frappe en premier c'est leur conscience aiguë des risques encourus par leurs enfants (échec scolaire, toxicomanie, délinquance, suicide, démoralisation, image négative d'eux-mêmes, chômage...). Les ménages apparaissent à la recherche constante du maintien de leurs équilibres constitutifs (dans les domaines économique, affectif, moral et social). Leur vie quotidienne est faite d'une succession de résolution de problèmes.

Le projet de cette recherche fondé sur la description des actions menées par ces familles pour lutter contre la précarité a encouragé des familles à accepter de participer à ce travail d'enquête, à ouvrir leurs portes, à montrer leurs fiches de paye, de RMI, d'allocations familiales, de pension. Elles n'ont pas caché non plus leurs difficultés, leurs espoirs et leurs combats.

\section{Présentation des familles et de leur cadre de vie}

Vingt des trente familles interrogées dans le cadre de cette enquête sont maghrébines et franco maghrébines. Il y a également deux familles espagnoles et une congolaise. Dans onze de ces familles, les parents ont mené des démarches de naturalisation (abouties ou en cours, refusée à une personne); les enfants de leur côté ont opté ou opteront pour la nationalité française à leur majorité. Sur les trente familles, on compte cent onze enfants.

\footnotetext{
4. Jazouli A., Les années banlieues, Paris, Seuil, 1992.

5. Delcroix C. avec la participation de Bertaux D. et Saint Martin C., Rohi D. Zegnani S., Enjeux prioritaires et types de conduites des familles populaires face à la précarité, CADIS, décembre - recherche financée par la Caisse Nationale d'Allocations Familiales en réponse à l'appel d'offre Précarité, trajectoires, modes de vie, 1998.

6. On ne peut parler des familles qui ont refusé les entretiens. Celles qui ont été rencontrées suffisent à contredire le stéréotype.
} 
Elles habitent dans des logements sociaux situés dans la cité de Bagatelle à Toulouse ${ }^{7}$ et ce depuis de nombreuses années (vingt-deux depuis plus de dix ans et près de la moitié d'entre elles depuis plus de vingt ans).

Les parents sont souvent analphabètes $(1 / 3)$ ou très peu scolarisés (niveau primaire), les femmes plus encore que les hommes. Certains ont suivi des études qui se sont terminées au collège et pour quatre cas au lycée, tandis que plus de la moitié des pères ont poursuivi des formations professionnelles allant du CAP au BEP professionnel.

De nombreux enfants sont en cours de scolarité (primaire, collège, école professionnelle, université). Certains d'entre eux ont atteint avec succès la voie des études supérieures (3 familles), la majorité poursuit des formations de type professionnel (CAP, BEP). Plusieurs (six) ont beaucoup de mal à trouver des patrons qui acceptent de les prendre comme apprentis, ou même de trouver un stage qui est cependant obligatoire pour obtenir le diplôme. Dix jeunes ont quitté l'école à 16 ans sans diplôme. Dans six familles certains jeunes ont connu des épisodes marqués par des problèmes d'exclusion scolaire temporaire, de placement dans des institutions spécialisées, de délinquance (vols, rodéos, agression de type bagarres, incivilités...). Pendant le temps de cette enquête, une famille a même perdu un fils au cours d'un accident de voiture survenu dans le cadre d'un rodéo. Dans le quartier de Bagatelle où nous avons travaillé, plus d'une dizaine de jeunes n'appartenant pas aux familles interviewées sont morts dans des conditions violentes.

La majorité des pères de famille sont venus des campagnes du Maghreb, d'Espagne et de France pour travailler comme ouvriers dans le bâtiment, les travaux publics, les transports routiers, urbains et interurbains (chauffeurs, livreurs, camionneurs) et le nettoyage industriel. Dix-sept d'entre eux travaillent et perçoivent le SMIC. Quatre sont au chômage, quatre sont en arrêt maladie, deux sont retraités et un est en formation.

La moitié des mères de famille travaillent dans le secteur du nettoyage des bureaux et de l'aide aux personnes. Elles ont des statuts professionnels précaires (CES, CDD) et occupent des emplois à temps partiels, peu rémunérés.

Les accidents du travail touchent treize pères, soit près de $50 \%$ d'entre eux (certains d'entre eux en ont subi plusieurs). Six personnes, dont une femme, sont victimes de maladies professionnelles.

Sur le plan des revenus, la population adulte des familles étudiées constitue une population de travailleurs mal payés (working poor) c'est-à-dire des personnes qui pour la plupart sont au travail ou en recherche de travail mais dont les revenus (en général des salaires) sont néanmoins si bas qu'elles se retrouvent, économiquement parlant, à peu près au même niveau de bas revenu que les assistés. Il ne s'agit pas de pauvres au sens (sociolo- gique) d'assistés sociaux mais bien de travailleurs recevant des salaires très bas. La faiblesse de leurs salaires est le reflet de la situation conjoncturelle du marché du travail, qui compte tenu du chômage de masse permet aux employeurs de payer très mal leurs employés.

D'après nos calculs les revenus des familles s'échelonnent de $2500 \mathrm{~F}$ à un peu plus de $5500 \mathrm{~F}$ par unité de consommation ${ }^{8}$. Nous avons distingué trois groupes de familles :

- celles qui ont des revenus par unité de consommation inférieurs à $3500 \mathrm{~F}$ (en 1998) peuvent être qualifiées de "très bas revenus"; ce que les organisations internationales appellent "sous le seuil de pauvreté"

- celles qui ont des revenus par unité de consommation situés entre $3500 \mathrm{~F}$ (environ) et $4500 \mathrm{~F}$ peuvent être qualifiées de "bas revenus"; rappelons que cette ligne de bas revenus se situe, dans la France de 1999, à environ $4000 \mathrm{~F}$ par unité de consommation (la moitié du revenu médian par u. c., soit $7800 \mathrm{~F}$ en 1998);

- et enfin celles qui ont des revenus par unité de consommation supérieurs à $4500 \mathrm{~F}$.

L'étude fine de leurs budgets et leur comparaison nous a permis d'observer des différences dans les structures de consommation liées aux préoccupations des parents pour assurer le devenir de leurs enfants.

\section{Éduquer en cité, c'est prévenir les risques}

Pour les parents que nous avons rencontrés, l'éducation de leurs enfants doit entre autre chose répondre à l'obligation d'apparaître «comme intégrée». En effet, à

7. Au sein de I'agglomération urbaine de Toulouse le quartier de Bagatelle possède une identité forte due à l'unité architecturale de son bâti (c'est un quartier qui comme beaucoup de cités HLM en France, a été construit d'un seul coup), à la relative homogénéité des ressources économiques et culturelles des familles qui y résident (il s'agit pour l'essentiel de logements sociaux), à son histoire spécifique. A ces caractéristiques objectives, il convient malheureusement d'ajouter l'image souvent exagérément négative qu'en donnent les médias. Bagatelle ne constitue pas seulement un espace urbain mais un véritable espace social : un territoire constitué en lieu de vie où les habitants vivent et échangent. C'est aussi une zone d'intervention des autorités administratives : le quartier est pris en compte depuis 1995 par le dispositif contrats-ville, il existe une zone d'éducation prioritaire; un nombre élevé de services sociaux et d'associations y sont présents et actifs. Pour mieux connaître les conditions de vie des habitants de Bagatelle et de quartiers semblables à travers toute la France voir aussi Collectif, En marge de la ville : ces quartiers dont on parle, Paris, L'Aube, 1998.

8. Pour tenir compte des économies d'échelle, on calcule le revenu par unité de consommation en attribuant des poids différents à chaque membre du ménage : le père compte pour 1 , la mère compte pour 0,5 , chaque enfant compte pour 0,3 jusqu'à 14 ans et 0,5 au-delà. Une mère chef de famille compte pour 1,2. Par exemple : pour une famille de 4 enfants (dont deux adolescents) ayant un revenu total de $14000 \mathrm{~F}$, le revenu par unité de consommation sera de $14000 \mathrm{~F} /(1+0,5+2 \times 0,5+2 \times 0,3)=3,1$ ce qui fait $4516 \mathrm{~F}$ par unité de consommation (soit un revenu juste au-dessus de la ligne des bas revenus). Voir Broilliard C., Le Jeannic T., Oger P., «Une source, une méthode pour mesurer localement la pauvreté », Recherches et prévisions, n 52, 1998, 37-58. 
chaque étape de la vie familiale les parents sont soumis à un examen de leur degré d'intégration sur la base de critères économiques, culturels, politiques, religieux, sociaux (relationnels) toujours plus contraignants. C'est le cas dans toutes les démarches qu'ils entreprennent vis-à-vis des institutions (école, services sociaux, offices HLM... etc.). Un des critères de l'intégration est le comportement de leurs enfants; d'où l'importance pour eux de trouver des styles éducatifs conformes aux normes "françaises».

Leurs pratiques paraissent pouvoir se laisser classer, du point de vue des styles éducatifs et du style de vie plus généralement, en deux groupes et deux seulement : des pratiques de repli et de protection contre les risques (logique de défense); et des pratiques qui s'ouvrent vers l'extérieur mais avec un strict contrôle de cette ouverture (logique de passage).

\section{La logique de défense}

La logique de défense s'appuie sur une hiérarchie des priorités qui assurent l'équilibre familial. Il faut pouvoir nourrir ses enfants, payer le loyer et les charges. Il s'agit du minimum vital qui permet d'être en règle (dans d'autres milieux sociaux l'accent serait sans doute mis sur d'autres objectifs car celui d'être en règle serait atteint en quelque sorte naturellement). Une des conséquences de ces choix est le maintien d'une distance avec des voisins que l'on se refuse à fréquenter pour ne pas devoir les inviter et mettre en danger l'équilibre budgétaire précaire. Un père ( $M$. Ahmed) nous dit :

"Je connais beaucoup de gens et ne fréquente personne $[\ldots]$ Il vaut mieux être grand chez soi que petit chez les autres».

Dans les familles les plus pauvres les choix budgétaires portent sur la volonté de payer régulièrement le loyer et d'être en règle en sacrifiant toute dépense non indispensable (essentiellement les loisirs). Éviter la saisie par l'huissier, conserver son logement sont autant de signaux donnés aux enfants de la capacité parentale à faire face aux difficultés liées à la précarité. C'est un des moyens privilégiés d'expliquer aux enfants le rapport à la loi.

«Au point de vue financier, je n'ai de l'argent que pour nous nourrir... Mes enfants ne comprennent pas. Ils regardent beaucoup la publicité. Ils demandent pourquoi on ne peut s'acheter ceci ou cela. La petite est proche de moi, elle comprend. Les enfants (ils sont quatre en tout) me disent "pourquoi on ne change pas de maison?» Je leur répète : l'important, c'est d'abord de payer le loyer" (Mme Fatima).

Ce manque de compréhension peut parfois amener l'enfant à une forme de violence : celle du vol.
Voici l'interprétation d'un père (famille Marcel) à propos du comportement de son fils :

"Je pense qu'il s'aperçoit qu'on ne peut pas lui offrir ce qu'il voudrait; d'ailleurs ce qu'il veut, on ne pourrait jamais lui payer car c'est vraiment trop cher, donc je pense que c'est un peu pour ça qu'il vole.»

Face à ces difficultés, les familles que nous avons rencontrées mettent en œuvre différents types de conduites. Ainsi, nous avons parlé avec un père qui

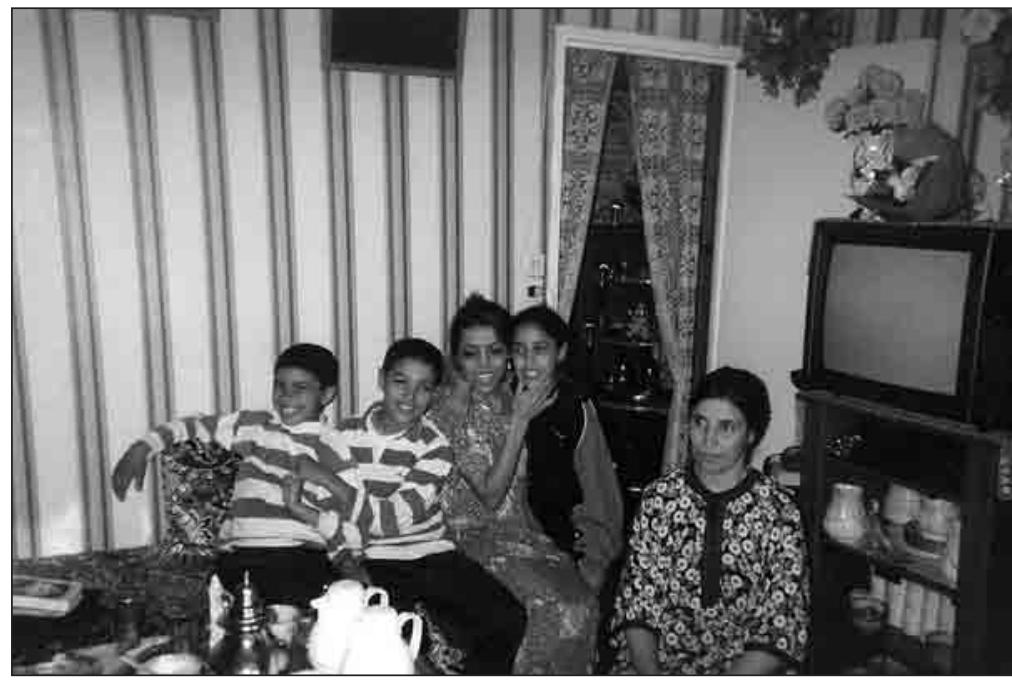

Une famille du quartier de Bagatelle à Toulouse.

conduit lui-même ses enfants à l'école. Étant accidenté du travail depuis de nombreuses années, il s'occupe d'acheter tout ce dont ils ont besoin pour l'école et pour s'habiller.

«Quand ils parlent de l'école les enfants, qu'est-ce qu'ils parlent, ils disent qu'il faut acheter des cahiers, des stylos... c'est obligatoire... les enfants, je m'en occupe, je les amène, je les ramène, je les amène, je les ramène» (M. Mohamed).

«Tu vois ce qu'ils font maintenant, maintenant c'est la drogue, il n'y a pas de travail, maintenant, c'est la drogue. Chez nous à l'époque (en Algérie et au Maroc), ça n'existait pas. Depuis le début des années 80 , la vie a beaucoup changé. Il y a trop de liberté... Les flics, ils attrapent les jeunes qui font des conneries. Ils les présentent au juge le matin qui les lâche le soir parce que les prisons sont pleines... Maintenant si tu engueules ton fils ou ta fille, ils vont déposer plainte. Ils vont venir me dire que vous n'avez pas le droit de taper votre fille... Tu laisses ta voiture dehors, ils vont la casser... Ils disaient à l'époque, tu voles un œuf, tu voles un bœuf. Maintenant, ils volent tout : un œuf, un bœuf, un poste...».

Pour éviter les dangers à ses enfants, il s'occupe de tout. Il les amène en vacances dans sa diesel 500. Parfois, il va avec eux au Parc de la Ramée. Sa femme ne 
parle pas français. Il ne souhaite pas qu'elle l'apprenne car il a peur qu'elle rencontre des personnes qui pourraient la détourner de sa famille.

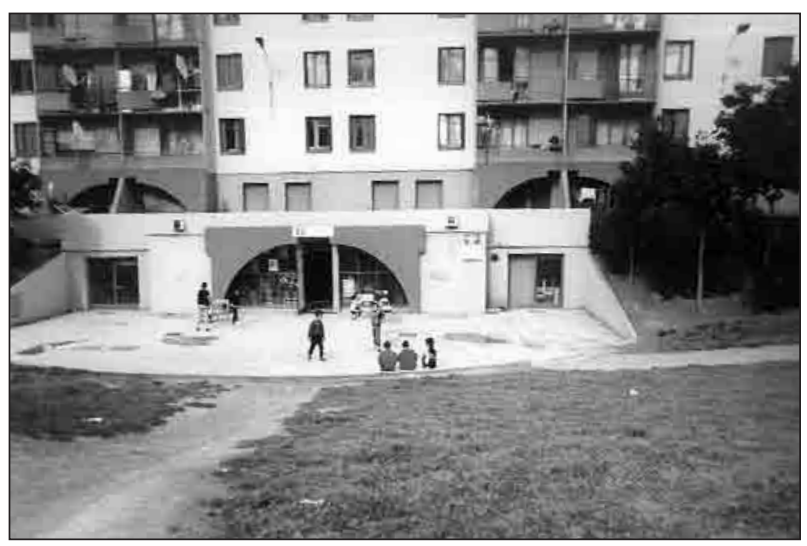

La cité de Bagatelle à Toulouse.

Cette crainte provient de la conscience du malentendu qui peut naitre de la rencontre entre des travailleurs sociaux et les familles immigrées (originaires du Maghreb).

"On est en présence de deux discours croisés qui ne se rencontrent pas. D'un côté, les travailleurs sociaux parlent au nom d'une rationalité moderne et de ses formes instituées, mais aussi d'une certaine morale de sauvegarde de l'enfance, de libération de la femme, d'un rôle hygiéniste, d'éducation... De l'autre, on pense être en présence de relations avec une famille élargie au quartier» ${ }^{\text {? }}$.

Dans les familles disposant d'un revenu un peu supérieur les (petits) moyens supplémentaires sont consacrés à éviter de trop fortes frustrations aux enfants, par exemple en matière vestimentaire (achat de vêtements «de marque»).

"J'ai mille francs par semaine pour faire manger dix personnes. Je passe deux heures tous les jours dans le marché pour obtenir les prix les moins chers. Je demande les prix, je retiens, je compare tout. Ça part très vite. Il faut faire face aux besoins des enfants. Ils veulent des marques. La semaine dernière, j'ai économisé sur les courses parce que (la semaine d'avant) Saïd (le garçon de 15 ans) voulait des chaussures de tennis de marque.

Il m'a dit : «maman, c'est pas plus cher!» Il les a mises dans le caddie. A la caisse, quand j'ai vu passer la paire : 450 F! J'avais compté 200 F! J'ai senti une grosse bouffée de chaleur. Il y avait du monde derrière... j'ai payé. Mais la semaine suivante, il a bien fallu rattraper...

Tous les jeudis il y a un marché de vêtements d'occasion pour les petits. J'y vais, je les habille comme ça. Il faut prévoir tout le temps, sinon...

Il faut préparer des cadeaux pour la fête de l'Aïd. Je commence deux mois avant, je les achète petit à petit, et je les cache». (Extrait d'entretien avec une mère de famille).
À travers ce témoignage on comprend qu'acheter à bon escient est un vrai travail. Le travail d'acheteuse d'une ménagère consiste à comparer non seulement les prix mais les valeurs d'usage. Mme K. ne se contente pas de rassembler une information maximale sur l'état du marché. Avant de prendre une décision d'achat elle réfléchit longuement aux arbitrages qu'elle doit faire entre des types de marchandises différentes (c'est-à-dire, en termes économiques, sur les valeurs d'usage ou "utilités" pour tel ou tel de ses enfants). Elle suit attentivement le développement du vécu de chacun d'eux; elle sait ce dont il rêve à chaque moment; elle essaie de deviner l'intensité de ce rêve, et quelle déception, démotivation, crise pourrait suivre sa non réalisation. Elle a compris que, malgré le manque d'argent, il faut de temps en temps savoir dépenser pour que l'enfant puisse satisfaire son souhait intime; elle connaît les dangers des frustrations trop nombreuses, trop intenses et trop longtemps cumulées; face au sentiment d'injustice que crée en chacun de ses enfants le décalage permanent entre désirs de consommation (créés, entretenus, magnifiés par la publicité) et les possibilités concrètes de les réaliser, elle peut seulement en surveiller de près la montée, essayer de sentir le moment où il risque de dépasser un certain seuil psychologique qui déboucherait sur une conduite agressive (ou auto-agressive), et intervenir juste avant pour désamorcer le danger.

\section{La logique de passage}

Dans le groupe des familles qui ont des moyens un peu plus élevés, le superflu est consacré à permettre aux enfants d'avoir plus d'activités à l'extérieur. L'objectif poursuivi par les familles qui appliquent ce style éducatif est d'aider leurs enfants à s'ouvrir sur le monde, c'est-à-dire permettre aux enfants de sortir du quartier pour rencontrer d'autres personnes. Il s'agit d'une ouverture contrôlée. Ils sont autorisés à partir dans le cadre scolaire ou associatif ou encore voyagent en France ou ailleurs en compagnie de leurs parents. Ils ont des activités de loisir qui se déroulent au sein des structures du quartier.

«Mes filles ont toutes été à la Bulle Rose. Celle-ci recevait les enfants de quatre à douze ans. (Cette structure aujourd'hui a changé de nom et de lieu). Elles y ont rencontré tous les enfants du quartier. Ils faisaient des sorties pour les enfants et parfois aussi avec les parents. On se rencontrait. C'était bien. Les sorties étaient très appréciées. Toutes les mères se rencontraient dans un autre milieu. Il y avait parfois aussi des pères, mais c'était très rare». (Mme Tahar)

9. Boulahbel-Villac Y., L'intégration au féminin, Espaces et familles, CNAF, n 16, mai, 1991. 
L'ouverture concerne aussi les parents. La voiture représente l'outil indispensable de cette rencontre avec l'extérieur. Elle est très importante car elle permet de s'échapper d'un lieu tel que Bagatelle. Elle donne les moyens de travailler.

"Un véhicule, c'est indispensable aujourd'hui pour aller travailler, pour faire des courses, pour bouger, c'est indispensable!» (fils de 24 ans, famille Lilla).

Pour ces parents, et en particulier pour les pères, leur fonction d'éducateur passe par une interrogation constante. En s'appuyant sur leurs capacités à comparer les situations, les lieux et les contextes, ils informent leurs enfants et leurs ouvrent des «champs de possibles».

Dans ces familles les sorties sont permises mais jusqu'à une heure précise. Elles sont considérées comme importantes pour l'équilibre et l'avenir des enfants. La négociation de ces normes n'est pas toujours explicite, elle passe souvent par le "non-dit» ${ }^{10}$. C'est par là qu'elles sont apprises et comprises.

Cette forme de socialisation est très fréquente dans les familles originaires du Maghreb. En effet, dans la mesure où le père représente la figure de l'autorité, discuter directement de la loi, la négocier pourrait remettre en cause son autorité. On parle souvent du silence des pères maghrébins et on l'interprète comme une absence de communication, une déficience d'éducation et même comme un effacement de la figure du père. C'est un contresens, qui ne tient pas compte de la spécificité d'un modèle éducatif reposant tout entier sur un exercice de l'autorité paternelle qui ne passe pas forcément par l'utilisation des mots pour se faire comprendre mais au contraire par d'autres formes de communication ${ }^{11}$.

On en aura une première idée si on pense à ce qu'étaient les figures de père dans les familles traditionnelles françaises jusqu'au début des années 60 . Dans ce modèle, toute l'autorité émane du père. Pour conserver à la fonction de père toute l'autorité qui lui est dévolue par sa culture, il faut qu'il garde une distance vis-à-vis de ses enfants, ne perde jamais la face devant eux, ne se laisse arracher aucune décision, ne négocie jamais directement avec eux. C'est à la mère et aux frères et sœurs plus âgés que revient la responsabilité d'engager le dialogue avec les enfants.

Le silence ne signifie aucunement qu'il n'y ait pas de communication entre le père maghrébin et ses enfants. Celle-ci ne passe pas par des mots mais par des attitudes exprimant par exemple la réprobation ou l'approbation, la fierté ou son contraire. Plus la figure d'autorité est forte et plus les signes non verbaux prennent du poids. De plus cette distance se trouve souvent renforcée au moment de l'entrée dans l'adolescence. Elle est particulièrement ressentie par les filles.
«Avant je lui parlais plus qu’à ma mère mais quand j'ai eu quinze ans, il y a eu beaucoup de sujets tabous. Pour les sorties, par exemple, j'en parle à ma mère. C'est au moment de sortir, qu'il m'amène ou qu'il vient me chercher; mais de ce qui se passe à l'intérieur, on n'en parle pas. Il sait les choses mais il ne me demandera pas comment ça s'est passé. C'est du non-dit. Quand on ne peut plus lui parler, on a parfois l'impression de ne plus être comprise, mais en fait c'est du non-entendu». (fille de dix-sept ans, famille Tahar).

La possibilité de donner à ses enfants une éducation ouverte sur le monde extérieur est considérée par un certain nombre de pères comme une expérimentation qui demande beaucoup d'attention et de réflexion. La comparaison leur apparait comme essentielle, surtout quand il s'agit de savoir quel espace de liberté donner.

"Tous les enfants ne sont pas élevés de la même manière. C'est différent oui, la différence, elle est flagrante. Ici, il y a beaucoup plus de liberté. Tout est de leur côté. Là-bas (en Algérie), ils ne sont pas élevés aussi librement. Pourtant là-bas, les enfants ne sont pas moins heureux. Ici, c'est un peu trop. Ils font ce qu'ils veulent et uniquement ce qu'ils veulent. Le juste milieu, c'est pas évident. Quand on voit les collèges et les lycées, il s'y passe des choses incroyables. Quand on voit qu'un professeur n'a pas le droit de dire ce qu'il a envie de dire. Parfois, il se fait tabasser pendant la classe. Peut-être que làbas ils n'ont pas ce qu'il faut, mais ici ils en font peut-être un peu trop. Parfois on parle de ça avec les autres pères. Les gens sont conscients. Pour trouver des solutions, il faut le vouloir, il faut savoir le faire.» (M. Tahar)

Un des problèmes que rencontrent ces pères dans la gestion de leurs relations éducatives avec leurs enfants porte sur les interventions des institutions (travailleurs sociaux, magistrats). Celles-ci sont animées des meilleures intentions mais aboutissent parfois à l'effet inverse du résultat recherché : en voulant protéger soit l'épouse, soit les enfants de l'autoritarisme d'un père perçu comme décalé par rapport à la culture démocratique du pays d'accueil, ces interventions mettent en cause publiquement l'autorité du père sans se rendre compte qu'elle constitue la clef de voûte de ce type de famille et la référence symbolique de la loi pour les enfants.

L'exemple que nous donnons ici nous paraît très significatif : un père d'origine maghrébine s'aperçoit que sa fille de 14 ans sèche l'école depuis un mois. S'efforçant d'établir un strict contrôle de ses allées et

10. Zerdoumi N., Enfants d'hier, Paris, Ed. François Maspéro, 1979.

11. Sayad A., L'immigration ou les paradoxes de l'altérité, Bruxelles, Deboeck, 1997. 
venues, il est bientôt convoqué par la Justice (à laquelle sa fille est venue se plaindre), soupçonné de séquestration et menacé de se voir retirer sa fille. Pour autant il refuse de lâcher prise, étant persuadé de bien faire :

"Le juge m’a dit "votre fille a le droit de sortir». Je me suis dit si à 15 ans elle fait ce qu'elle veut, alors comme père, je plie bagage. Je me suis demandé : si ça leur était arrivé, au juge et à l'éducateur, ce qui m'est arrivé avec ma fille, auraient-ils agi autrement que moi ? Ils te disent «si tu n'es pas content, on a des foyers». Cette période de discussion intense avec les institutionnels et ma fille, ça a été l'enfer pour moi; c'était plus dur que le travail, plus dur que toute autre chose. A les entendre parler, c'est eux qui avaient raison. Quand enfin, j’ai réussi à leur expliquer, ils ont compris que je voulais l'empêcher de sécher les cours et tout s'est arrêté. Mais si vous laissez les choses aller, deux ans après c'est trop tard. Que ça se soit bien terminé, c'est très important pour tout le monde. Si j'essaie de faire tout mon possible, de me priver pour leurs études et si je suis déçu, c'est dur! Ils ne se rendent pas compte».

Interviewée indépendamment, sa fille nous a confirmé la version donnée par son père :

«... Ce sont mes parents qui m’ont réveillée; mon père m'a interdit de la voir (une amie qui séchait les cours et se droguait), de lui parler. Il m’a surveillée pour aller à l'école. Non ce n'était pas la joie. Ça a duré de mars à juin. C'est ce qui m'a permis de passer de $3 e$ en seconde. Au début, j'étais frustrée mais une fois arrivée en seconde, j'ai vu qu'ils avaient totalement raison parce que je vois ce qu'elle est devenue».

Parmi les pères, ceux qui ont choisi cette voie de l'ouverture contrôlée dans l'éducation semblent aussi être ceux qui exercent une certaine "puissance sociale» ${ }^{12}$.

Parmi les mères, et notamment dans les familles monoparentales, nous avons découvert également des stratégies éducatives fondées sur l'ouverture et la responsabilisation des enfants. Ainsi, cette mère réunionnaise qui délègue une part de l'organisation ménagère du foyer aussi bien à ses fils (10 et 12 ans) qu'à ses filles (8 et 14 ans).

"Ils m'aident beaucoup les enfants. Je leur ai appris qu'il fallait se débrouiller tout seul, prendre ses responsabilités. Les enfants des Îles se débrouillent seuls. Ils font tout eux-mêmes.»

Une autre de ces mères a choisi de payer à sa fille une école privée au centre de Toulouse. Elle souhaite que celle-ci développe des réseaux de relations qui lui permettent plus tard de poursuivre des études supérieures et de devenir médecin. Ce choix l'éloigne du quartier, de l'échec scolaire associé aux écoles de Bagatelle. Parallèlement, elle lui montre les aspects positifs du quartier : «sa convivialité, ses liens de solidarité; Bagatelle est un village où l'on apprend à vivre ensemble avec d'autres qui viennent d'ailleurs (autres cultures, autres religions, autres langues).» (Mme Rachel)

\section{Ressources éducatives et ressources matérielles}

Ces styles de pratiques éducatives ne relèvent pas seulement de modèles éducatifs différents, mais au moins autant de niveaux différents de ressources matérielles. Nous avons entendu plusieurs fois des parents décrire comment leurs pratiques éducatives ont évolué en fonction de leur situation matérielle.

Parmi les autres ressources que mettent en ouvre les parents dans leur action éducative figurent leurs ressources subjectives (par opposition aux ressources objectives, aussi appelées "capitaux" par Pierre Bourdieu) et notamment leurs ressources communicationnelles. La recherche a permis à ce propos de vérifier encore une fois l'hypothèse élaborée au cours d'une enquête de terrain précédente (à Nantes Nord) ${ }^{13}$, à savoir que dans les familles où les parents, et en particulier les pères, communiquent avec leurs enfants, leur racontent par où ils sont passés, partagent avec eux leurs expériences de vie et les leçons qu'ils en ont tirées (leur "capital d'expérience biographique spécifique»), les enfants semblent mieux préparés à réussir une insertion. Il faut aussi mentionner comme ressource éducative l'exemple qu'ils donnent à leurs enfants en se battant au quotidien pour gagner leur vie et celle de leur famille, faisant ainsi preuve de courage et de ténacité.

\section{Les effets d'un contexte dévalorisant}

Qu'ils soient d'origine étrangère ou française, les pères ont travaillé presque tous comme ouvrier pendant la majeure partie de leur vie active. Cela signifie bien entendu des conditions de travail éprouvantes, des risques d'accidents importants, des périodes de chômage et des bas revenus. Ce sont des travailleurs mal payés. Qui plus est, le contexte économique s'est considérablement durci ces dix dernières années pour ce type de travailleurs. Leur taux de chômage est très

12. Delapierre G., «La puissance sociale des pères», Bulletin de la société lyonnaise pour l'enfance et l'adolescence, Lyon, 1993, 20-23, définit la puissance sociale des parents et en particulier du père comme la rencontre de trois capacités : la capacité de participer à des réseaux de solidarité vivants, la capacité à intégrer l'information et la capacité à mettre en action des savoirfaire pour régler des problèmes.

13. APS de Nantes et Delcroix C., Une nouvelle approche de la prévention de la délinquance des jeunes maghrébins : le rôle social des pères, Paris, Agence pour le développement des relations interculturelles, collection Savoirs et Perspectives, 1995. 
supérieur à la moyenne et les salaires réels ont baissé (à titre d'exemple, après 15 ans de travail comme ouvrier qualifié payé $14000 \mathrm{~F}$ par mois, $\mathrm{M}$. Tahar a perdu son emploi en 1988 et n'a retrouvé qu'un emploi d'ouvrier dans le nettoyage industriel pour un salaire de 6000 F). Près de la moitié ont été victimes de graves accidents de travail ou de maladies professionnelles. Bien qu'il ne soit pas dans les habitudes de ces pères de se plaindre, leurs enfants sont conscients de ce qu'ils ont subi, d'où chez eux le désir d'aider leurs parents mais peut-être aussi de les venger. Ces sentiments ne sont pas nouveaux dans l'histoire ouvrière française mais ils sont peut-être exacerbés aujourd'hui, entre autre par le mépris latent du discours public à l'égard de tous ceux qui n'ont pas réussi.

Le niveau de revenu de ces familles est très bas, même en tenant compte de l'apport des transferts sociaux. Cela engendre pour les enfants des frustrations permanentes, d'autant plus vives que le modèle culturel dominant de la société française se centre de plus en plus sur la consommation. Celle-ci confere la clé du statut, voire de l'identité, en particulier chez des jeunes issus de familles sans patrimoine. L'invitation de la publicité à participer à la vie sociale par la consommation est une composante structurelle de l'économie de marché; dès lors il n'est pas étonnant que l'on se sente exclu si l'on ne peut pas consommer.

L'école représente une chance de réussite (d'abord scolaire, personnelle, puis professionnelle, et enfin sociale) et certain(e) s parviennent à s'en saisir. Mais celui ou celle qui n'y parvient pas est conduit à se sentir personnellement responsable de son échec.

On trouve certes nombre d'enseignants qui cherchent à comprendre le contexte dans lequel vivent leurs élèves et qui s'efforcent d'adapter les formes, voire les contenus de leur enseignement à leurs classes. Mais il y a aussi les autres, et en particulier ceux qui sont animés de représentations à priori négatives envers les enfants des cités. C'est d'ailleurs l'une des difficultés des parents que de discerner la part de vérité quand un de leurs enfants explique ses mauvaises notes en assurant : «le professeur est raciste».

Le racisme est un réalité vécue, d'ailleurs bien plus complexe qu'on ne le dit puisqu'à un racisme ethnique se superpose un racisme de classe. Il se manifeste dans tous les compartiments de la vie sociale et parfois envahit l'institution scolaire. Par exemple dans de nombreuses filières scolaires (générales ou professionnelles), l'étudiant doit désormais trouver lui-même un stage en entreprise faute de quoi il ne peut obtenir le diplôme. Ceux qui n'auront pu se faire accepter, notamment du fait de leur origine ou de leur couleur, se voient aussi empêchés de terminer normalement leur cursus scolaire.

Quant aux marchés de l'emploi, ils sont, ce n'est plus un secret, marqués par les discriminations ${ }^{14}$.
Pour bien des jeunes, le seul espoir de trouver un travail est dans le secteur des emplois aidés (emplois jeunes, etc.). Il ne s'agit pas seulement des jeunes en échec scolaire mais d'une population beaucoup plus large, qui même si elle a des diplômes et la volonté farouche de faire ses preuves se trouve victime de discrimination. Or ce n'est en réalité qu'environ $20 \%$

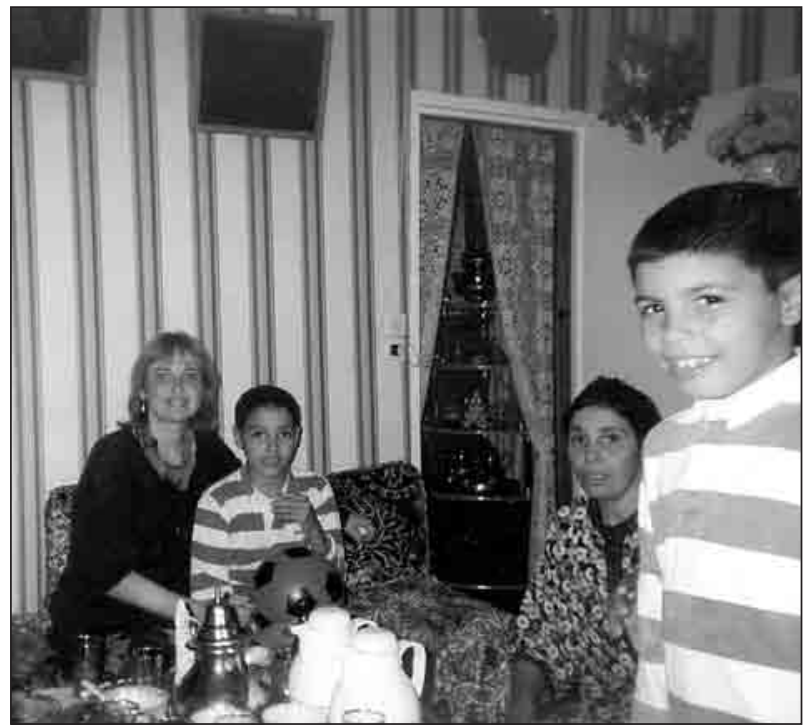

La chercheuse à la rencontre des familles de Bagatelle.

des emplois jeunes qui vont être attribués aux jeunes des cités. Dans leurs efforts de recherche de stages, de formation et d'emploi, ces jeunes essuient des successions de refus et d'humiliations qui peuvent conduire certains d'entre eux, en particulier les jeunes issus de l'immigration, à considérer qu'ils ne trouveront jamais ni emploi, ni place dans cette société.

\section{Dévaluation et agressivité}

Ce profond sentiment d'injustice ne trouve pas d'expression politique collective. Parfois il se manifeste brutalement à travers des émeutes, car beaucoup de jeunes des cités ont la conviction que les responsables politiques du pays portent plus d'attention aux revendications des catégories sociales qui se manifestent spectaculairement (agriculteurs ou camionneurs en colère) qu'aux autres. Mais comme le dit Saïd Bouamama $^{15}$, "ce qui est remis en cause (par les jeunes)

14. Tripier M., De Rudder V. et Vourc'h F., De la prévention du racisme dans I'entreprise en France, Paris, coédition CNRS et Université de Paris VII, 1995; Bataille P., Le racisme au travail, Paris, La Découverte, 1997.

15. Bouamama S., "Jeunesse, autorité et conflit », Paris, Migrants-formation $n^{\circ} 112$, mars 1998, 26-39.

16. Bui Trong L., «Les rapports des jeunes avec l'autorité policière », Paris, Migrants-formation $n^{\circ} 112$, mars 1998, 49-61. 
c'est un pouvoir donné, portant une autorité précise, vécue comme injuste; ce n'est en aucun cas le principe même de la loi et d'autorité... si les comportements des jeunes interpellent le concept de citoyenneté, c'est justement qu'ils posent la question de leur place sociale et celle du partage du pouvoir.»

Cette révolte qui ne trouve pas les moyens légaux de faire reconnaître l'existence d'une demande est parfois interprétée de manière erronée comme étant une violence fondée sur la haine et le « rejet global des institutions" 16 alors qu'elle n'est qu'un appel, un moment clef correspondant à une demande désespérée de prise en considération et de participation effective à la société. C'est ainsi que les cinq jeunes qui sont morts au cours d'un rodéo pendant notre enquête n'étaient pas des jeunes dépourvus de diplôme mais plutôt des garçons persuadés qu'ils ne parviendraient pas à trouver du travail et enclins, du même coup, à flirter avec la mort. Une mère de famille a constaté, en écoutant à sa fenêtre les conversations des jeunes qui stationnaient au bas de son immeuble, que ceux-ci décidaient de s'engager dans des rodéos après s'être rendus, durant la journée, à plusieurs entrevues d'embauche et avoir vécu des moments d'humiliation et de rejet.

L'intériorisation par les jeunes de l'image négative qui leur est renvoyée par la société est sans aucun doute l'une des difficultés les plus graves auxquelles les parents, mais aussi les professionnels de l'éducation et du social, sont confrontés. Cette intériorisation commence bien souvent dès la maternelle. C'est ainsi qu'une petite fille de quatre ans est venue dire à l'une des enquêtrices de notre recherche «tu es Arabe, toi? Parce que moi je le suis mais je ne veux pas le dire parce que ce n'est pas bien d'être Arabe». Ce problème amène des parents et des professionnels à se rapprocher pour combattre dès la petite enfance ce processus de dévalorisation qui peut conduire assez vite les enfants devenus adolescents à poser des conduites de violence. C'est ainsi qu'un professionnel du social fondateur de Bagamômes (lieu, appartenant à la CAF, qui reçoit des enfants de six à douze ans), souligne l'importance d'une proximité et d'une mise en commun du vécu entre enfants, familles et éducateurs. Pour lui, "les enfants des quartiers défavorisés ont une caractéristique commune : ils présentent une certaine vulnérabilité sociale, culturelle et économique. Ils mettent de la bonne volonté (exemple des soutiens scolaires très fréquentés) pour s'intégrer socialement. Mais à la suite d'échecs successifs, de dévalorisation, d'images négatives d'eux-mêmes, leur bonne volonté se change progressivement en ressentiment. Ils adoptent des attitudes de défense, de mutisme, de ruse, aussi d'agression... Cet état de désengagement et de non participation peut mener à la dissociabilité et à la délinquance» ${ }^{17}$.

\section{Les parents ne doivent pas être les boucs émissaires}

La dénonciation de l'incapacité éducative des parents a tenté de s'imposer comme une idée reçue à force d'être répétée comme une évidence. Elle apparaît cependant, à qui s'approche du terrain, comme un pur et simple phénomène idéologique de désignation commode d'un bouc émissaire à la vindicte générale. Notre enquête montre au contraire que de nombreux parents des cités font des efforts éducatifs considérables. Elle montre également que les enfants qui ont certains types de comportements déviants, tels que les rodéos et les émeutes, se recrutent aussi dans ces familles attentives à l'éducation de leurs enfants.

C'est par référence à la dureté du contexte que l'on peut comprendre pourquoi les jeunes de ces cités agissent comme ils le font. Comprendre les motivations de ces actes ne signifie pas les absoudre mais constitue un premier pas dans l'élaboration de solutions efficaces, qui passent notamment par la participation des parents ${ }^{18}$.

La société française a tort de considérer les habitants des cités de banlieues comme des exclus, des classes dangereuses, des ennemis. Elle devrait au contraire les prendre, qu'ils soient adultes, jeunes ou enfants, comme des alliés potentiels.

\section{Catherine Delcroix}

\footnotetext{
17. Garcia S., Bagamômes, un lieu à vivre, Toulouse, IRFCES Saint-Simon, juin, 1998.

18. Une manière d'encourager les parents à exercer leurs responsabilités éducatives en collaboration avec les professionnels du social et de l'éducation peut, par exemple, passer par la reconstitution de leurs histoires de famille avec l'appui de travailleurs sociaux. Cette reconstitution se fait à partir de «récits croisés» (Delcroix, 1995). Ce type d'expérience a déjà été mené par des travailleurs sociaux d'un club de prévention à Nantes Nord (APS de Nantes et Delcroix Catherine 1995). II a permis une remise en question des à priori des travailleurs sociaux concernant les parents, et en particulier les pères maghrébins, qui ne se soucieraient pas de leur progéniture. En réalité, beaucoup de pères maghrébins s'intéressent de près à leurs enfants adolescents. Ils vont jusqu'à s'organiser en association pour essayer de leur trouver du travail, des activités de loisir et pour avoir les moyens d'assurer leur défense en cas de problème avec la police. Suite à ce travail, ils se sont déclarés prêts à collaborer avec les travailleurs sociaux pour favoriser la prévention de la délinquance et plus largement la prévention des risques encourus par leurs enfants sur le quartier. Ils se sont sentis reconnus comme des parents responsables par les travailleurs sociaux. Cette reconnaissance a également permis aux jeunes de mieux connaître l'histoire de leur famille et de comprendre les projets et les attentes parentales à leur égard. Cette histoire familiale, non légitimée parce qu'étrangère et ouvrière, avait parfois été tue. A la suite de cette expérience et de ses conséquences, les familles ouvrières françaises de la cité ont elles aussi demandé à participer à cette enquête.
} 


\section{BIBLIOGRAPHIE}

Livres - rapports

APS de Nantes et Delcroix C., Une nouvelle approche de la prévention de la délinquance des jeunes maghrébins, le rôle social des pères, Paris, ADRI, coll. Savoirs et Perspectives, 1995.

Bataille P., Le racisme au travail, Paris, La Découverte, 1997.

Bordet J., Les «jeunes de la cité», Paris, Seuil, 1998.

Collectif, En marge de la ville : ces quartiers dont on parle, La Tour d'Aigues, L'Aube, 1998.

Delcroix C., avec la participation de Bertaux D. et Saint-Martin C., Rohi D., Zegnani S., Enjeux prioritaires et types de conduites de familles populaires face à la précarité, Paris, CADIS, CNAF, 1998.

Garcia S., Bagamômes, un lieu à vivre, Toulouse, IRFCES SaintSimon, 1990.

Jazouli A., Les années banlieues, Paris, Seuil, 1992.

Leger A., Tripier M., Fuir ou construire l'école populaire, Paris, Méridiens Klincksieck, 1988.

Lepoutre D., Cour de banlieue, codes, rite et langages, Paris, Odile Jacob, 1997.

Sayad A., L'immigration ou les paradoxes de l'altérité, Bruxelles, De Boeck, 1997.
Tripier M., De Rudder V., Vourc'h F., De la prévention du racisme dans l'entreprise en France, Paris, CNRS et Université de Paris VII, 1995.

Zerdoumi N., Enfants d'hier, Paris, François Maspéro, 1979.

Articles

Bouamama S., "Jeunesse, autorité et conflit», Paris, MigrantsFormation, $\mathrm{n}^{\circ} 112$, 1998, 26-39.

Boulahbel-Villac Y., "L'intégration au féminin», Paris, Espaces et familles, CNAF, n 16, 1991.

Broilliard C., Le Jeannic T., Oger P., «Une source, une méthode pour mesurer localement la pauvreté», Paris, Recherches et prévisions, $\mathrm{n}^{\circ} 52,1998,37-58$.

Delapierre G., "La puissance sociale des pères », Lyon, Bulletin de la Société lyonnaise pour l'enfance et l'adolescence, 20-23, 1993.

Delcroix C., "Des récits croisés aux histoires de familles", Londres, Current sociology/La sociologie contemporaine, $\mathrm{n}^{\circ} 43$, 1995, 61-67.

Fize M., "L'autorité maltraitée... et revendiquée», Paris, Migrants-Formation, $\mathrm{n}^{\circ}$ 112, 1998, 20-25.

Wacquant L., "De l'État social à l'État pénal», Actes de la recherche en sciences sociales, $n^{\circ} 124$, septembre 1998, 5-15.

Catherine Delcroix, sociologue au Laboratoire Printemps (Université de Versailles Saint-Quentin en Yvelines), associée au Laboratoire Cadis-CNRS/EHESS a dirigé la recherche pour la Caisse nationale d'Allocations familiales, gérée par le CADIS : Enjeux prioritaires et types de conduites des familles face à la précarité. Elle a publié aussi : Médiatrices dans les quartiers fragilisés : le lien, Paris, La Documentation française, 1996, et «L'immigration, histoires et mémoires, la présence des pères », Paris, Informations sociales, $n^{\circ} 55,1996,32-42$. 